\title{
Cellulose Tape Method
}

National Cancer Institute

\section{Source}

National Cancer Institute. Cellulose Tape Method. NCI Thesaurus. Code C85578.

A microbiological technique for specimen collection and mounting for microscopic examination that utilizes a cellulose-based adhesive ribbon. 